\title{
A NEW TREATMENT OF THE LIFTING-LINE WING THEORY, WITH APPLICATIONS TO RIGID AND ELASTIC WINGS*
}

\author{
BY \\ W. R. SEARS \\ Cornell University
}

1. Introduction: the Prandtl airfoil theory. The approximate theory of three-dimensional airfoils known as the "lifting-line" theory was formulated in 1918 by L. Prandtl." It has been in general use since then, and a number of different techniques have been developed for the numerical solution of the equations involved. Notable among these are the methods of Glauert, ${ }^{2}$ Lotz, ${ }^{3}$ and Multhopp. ${ }^{4}$ The first two of these are based upon the use of trigonometric series, while the third involves solution of an integral equation by successive numerical approximations. In the present treatment, the Prandtl theory is formulated in terms of an integral equation of a classical type, and a practical solution in terms of its eigenfunctions is carried out. It is believed that certain advantages are offered by this procedure.

In the Prandtl theory the wing is assumed to be at rest in an incompressible fluid of infinite extent, whose velocity is uniform far upstream. The effects of viscosity are neglected, except as they are required to explain the presence of circulation about the wing. The wing is then replaced by a single vortex filament, or "lifting line", having at any spanwise station a strength equal to the circulation about a contour enclosing the wing at that station. There extends downstream from the lifting line a discontinuity surface, called the trailing-vortex sheet; hence the region is multiply connected. It is assumed that all the velocity components induced by the vortex filament and the vortex sheet are small in comparison with the undisturbed stream velocity; the vortex sheet may then be assumed to be approximately plane and to lie in the direction of the undisturbed velocity vector, at least for purposes of calculating certain induced velocity components at the lifting line.

The remaining approximation of the Prandtl theory is required to relate the circulation $\Gamma$ about any section of the wing to the geometry of the wing at that section and the induced velocity at the same point on the lifting line. Let us select a coordinate system such that the distance along the span is denoted by $y$, as indicated in Fig. 1; let $U$ denote the undisturbed stream velocity, $c(y)$ and $\alpha(y)$ the chord and angle of attack of the wing at $y$, and $w(y)$ the $z$ component of the induced velocity at point $(0, y, 0)$ on the lifting line. The relation assumed by Prandtl, by analogy with the production of circulation about an airfoil in two-dimensional flow, is

$$
\Gamma=\frac{1}{2} U c m\left(\alpha-\frac{w}{U}\right),
$$

where $m(y)$ is a number that is characteristic of the airfoil profile at $y$, namely the slope of the lift-coefficient curve for two-dimensional flow.

The most important practical problem of three-dimensional airfoil theory is the determination of $\Gamma(y)$ when the geometrical properties $\alpha, c$, and $m$ are prescribed functions of $y$; this requires the calculation of $w(y)$ in terms of $\Gamma(y)$. The strength of the vortex sheet, which according to the approximations stated above does not vary

*Received Nov. 28, 1947. 
with $x$, is given by $-d \Gamma / d y$. Prandtl calculated $w$ by integrating over the sheet; however, a treatment that is more convenient here is the one employed by Trefftz, ${ }^{5}$ who considered conditions in a transverse plane far downstream where the effects of the lifting line are negligible. In such a plane the induced flow is steady and two-dimensional, and by symmetry the induced velocity components in the $y$ and $z$ directions are twice as great as at the corresponding points in the plane of the lifting line.

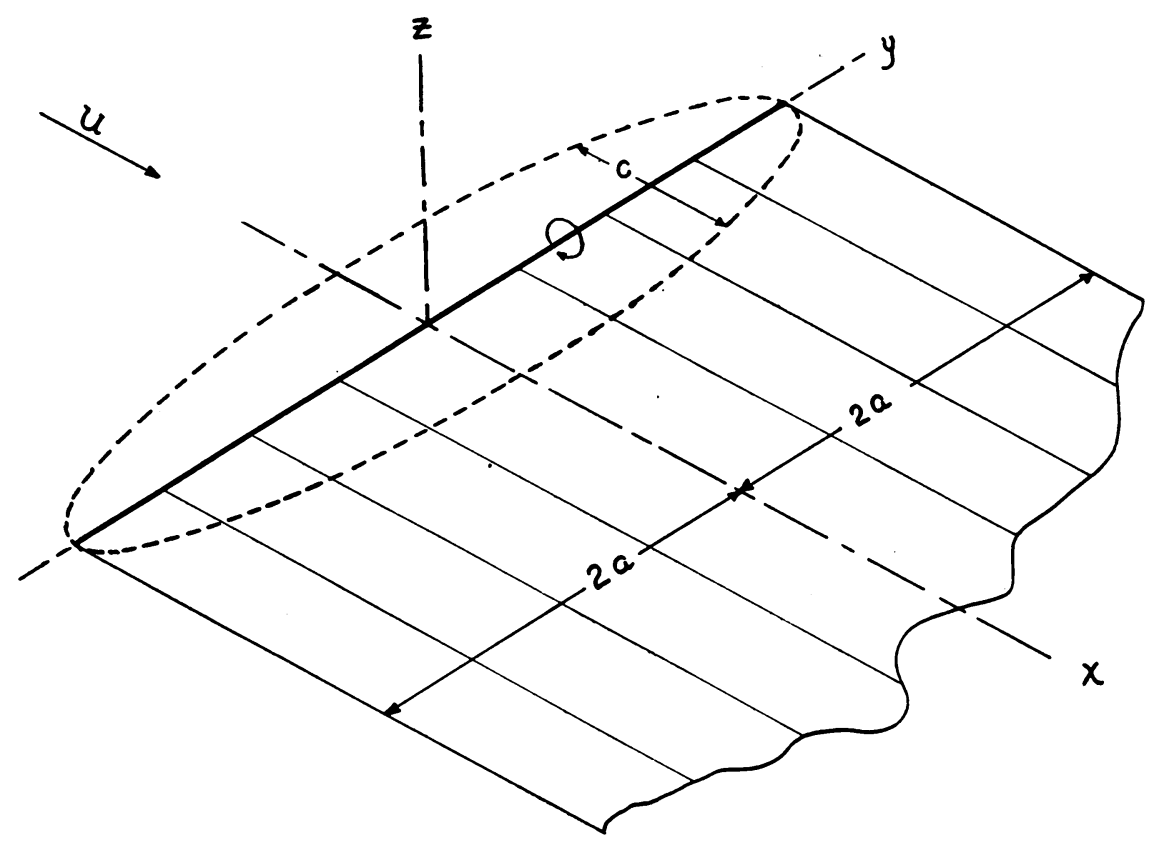

Frg. 1. Diagram showing notation.

Let $\varphi(y, z)$ be the velocity potential of the induced flow in Trefftz' plane. The jump in the value of $\varphi$ at the discontinuity surface is seen to be equal to $\Gamma(y)$; i.e.

$$
\begin{aligned}
\Gamma(y) & =\varphi(y,+0)-\varphi(y,-0) \\
& =2 \varphi(y), \text { say. }
\end{aligned}
$$

Hence the problem represented by Eq. (1) can be replaced by the following two-dimensional boundary-value problem:

$$
\begin{gathered}
\Delta \varphi \equiv \frac{\partial^{2} \varphi}{\partial y^{2}}+\frac{\partial^{2} \varphi}{\partial z^{2}}=0 \\
\text { (except on the slit } z=0, \quad-2 a \leqq y \leqq 2 a) \\
2 \varphi=\frac{1}{2} U c m\left(\alpha+\frac{1}{2 U} \frac{\partial \varphi}{\partial z}\right), \quad \text { for } \quad z=+0, \\
=-\frac{1}{2} U c m\left(\alpha+\frac{1}{2 U} \frac{\partial \varphi}{\partial z}\right), \quad \text { for } \quad z=-0
\end{gathered}
$$


It should be noted that, whereas the velocity potential has equal and opposite values on the two sides of the discontinuity surface, the $z$ component of the velocity is continuous at this surface.

2. Formulation as an integral equation. It is convenient at this point to employ the transformation

$$
y+i z=a\left(\zeta+\zeta^{-1}\right)
$$

by which the region exterior to the trace of the vortex sheet is mapped on the region outside the unit circle in the $\zeta$ plane. Here $4 a$ denotes the span of the wing. If $\zeta \equiv r e^{i \theta}$, the second and third of Eqs. (3) are replaced by the equations

$$
\left.\begin{array}{rl}
2 \varphi & =\frac{1}{2} U c m\left(\alpha+\frac{1}{4 a U \sin \theta} \frac{\partial \varphi}{\partial r}\right), \\
\varphi(\theta) & =-\varphi(2 \pi-\theta),
\end{array}\right\} \quad \text { for } r=1, \quad 0 \leqq \theta \leqq 2 \pi
$$

or, after rearrangement of terms,

$$
\left.\begin{array}{rl}
4 \frac{\sin \theta}{c m} \varphi-\frac{1}{4 a} \frac{\partial \varphi}{\partial r} & =U \alpha \sin \theta, \\
\varphi(\theta) & =-\varphi(2 \pi-\theta),
\end{array}\right\} \quad \text { for } r=1, \quad 0 \leqq \theta \leqq 2 \pi .
$$

If we introduce the notations

$$
\left.\begin{array}{rl}
f(\theta) & \equiv \frac{(c m)_{0}}{c m} \sin \theta=f(2 \pi-\theta), \\
g(\theta) & \equiv U(c m)_{0} \alpha \sin \theta=g(2 \pi-\theta),
\end{array}\right\} \quad 0 \leqq \theta \leqq 2 \pi
$$

where $(\mathrm{cm})_{0}$ is the value of the product $\mathrm{cm}$ at a convenient reference station, say at $y=0$, then we have the boundary-value problem in the form

$$
\begin{gathered}
\Delta \varphi=0 \quad \text { for } \quad r>1, \\
4 f \varphi-l \frac{\partial \varphi}{\partial r}=g \quad \text { for } \quad r=1, \\
\varphi(\theta)=-\varphi(2 \pi-\theta) .
\end{gathered}
$$

Several procedures have been suggested for the numerical solution of this nonhomogeneous boundary-value problem of the third kind. Lotz ${ }^{3}$ used trigonometric expansions of the functions $\varphi, f$, and $g$; this leads to an infinite set of non-homogeneous linear equations for the Fourier coefficients of $\varphi$. This set is different for each choice of $f$ and $g$, i.e. for each wing planform and angle-of-attack distribution. Schröder ${ }^{6}$ and Gebelein ${ }^{7}$ have set up the integral equation corresponding to the boundary-value problem, and Schröder has proposed a solution in terms of its eigenfunctions.

In the present treatment we formulate the integral equation by a somewhat different process, and carry out the practical solution in terms of its eigenfunctions. It appears 
that this recourse to the underlying mathematical character of the problem leads to certain essential simplifications, since the eigenfunctions and eigenvalues are characteristic of a planform $(f)$-in fact, of a family of planforms - and do not depend upon the angle-of-attack distribution $(g)$.

In view of the antisymmetrical character of the problem, Eq. (6) can immediately be replaced by an integral equation of the second kind by use of the Poisson equation for this case:

$$
\begin{aligned}
\varphi(\theta)= & -\frac{1}{\pi} \int_{0}^{2 \pi} \log \left|\csc \frac{\theta-\tau}{2}\right| \frac{\partial \varphi}{\partial r} d \tau \\
= & -\frac{4}{\pi l} \int_{0}^{2 \pi} \log \left|\csc \frac{\theta-\tau}{2}\right| f(\tau) \varphi(\tau) d \tau \\
& +\frac{1}{\pi l} \int_{0}^{2 \pi} \log \left|\csc \frac{\theta-\tau}{2}\right| g(\tau) d \tau .
\end{aligned}
$$

If we multiply both sides of Eq. (7) by $[f(\theta)]^{1 / 2}$ and denote $f^{1 / 2} \varphi$ by $\Phi$, we obtain a new integral equation involving a symmetrical kernel $K(\theta, \tau)=K(\tau, \theta)=$ $\log |\csc (\theta-\tau) / 2|[f(\theta) f(\tau)]^{1 / 2} ;$ i.e. the equation

$$
\Phi(\theta)=\lambda \int_{0}^{2 \pi} K(\theta, \tau) \Phi(\tau) d \tau+F(\theta)
$$

where we have also written $-4 / \pi l \equiv \lambda$ and

$$
F(\theta) \equiv \frac{1}{\pi l} \int_{0}^{2 \pi} K(\theta, \tau) \frac{g(\tau)}{[f(\tau)]^{1 / 2}} d \tau
$$

3. Solution of the integral equation. This integral equation [Eq. (8)] is of a classical type, having a real, symmetrical kernel of bounded integral-square, and there are available for its solution the theories of E. Schmidt, D. Hilbert, and others.* The solution of Eq. (8) is as follows:

$$
\Phi(\theta)=F(\theta)+\lambda\left\{\frac{F_{1}}{\lambda_{1}-\lambda} \Phi_{1}(\theta)+\frac{F_{2}}{\lambda_{2}-\lambda} \Phi_{2}(\theta)+\cdots\right\},
$$

where $\lambda_{1}, \lambda_{2}, \cdots$ are the eigenvalues and $\Phi_{1}(\theta), \Phi_{2}(\theta), \cdots$ the corresponding normalized eigenfunctions of the homogeneous integral equation

$$
\Phi_{i}(\theta)=\lambda_{i} \int_{0}^{2 \pi} K(\theta, \tau) \Phi_{i}(\tau) d \tau ; \quad i=1,2, \cdots
$$

and the definition of $F_{1}, F_{2}, \cdots$ is given by

Moreover,

$$
F_{i} \equiv \int_{0}^{2 \pi} F(\theta) \Phi_{i}(\theta) d \theta ; \quad i=1,2, \cdots
$$

(ii) the homogeneous equation [Eq. (10)] has infinitely many real eigenvalues, and, since the kernel $K(\theta, \tau)$ can be shown to be positive-definite, ${ }^{* *}$ all of its eigenvalues are

*See, for example, Reference 8, pp. 527-535, also Reference 9, pp. 104-118.

**Reference 9, p. 105. 
positive;

(iii) the eigenfunctions of the homogeneous equation are real functions of $\theta$ in the interval $0 \leqq \theta \leqq 2 \pi$, and constitute a complete system; they possess the property of orthogonality:

$$
\int_{0}^{2 \pi} \dot{\Phi}_{i}(\theta) \Phi_{j}(\theta) d \theta=\delta_{i j}
$$

(iv) any arbitrary function $y(\theta)$ can be represented in the interval $0 \leqq \theta \leqq 2 \pi$ by the series

where

$$
y(\theta)=c_{1} \Phi_{1}(\theta)+c_{2} \Phi_{2}(\theta)+\cdots,
$$

$$
c_{i} \equiv \int_{0}^{2 \pi} y(\theta) \Phi_{i}(\theta) d \theta ; \quad i=1,2, \cdots
$$

and the series (13) is absolutely and uniformly convergent in the interval; provided that $y(\theta)$ can be represented in the form

$$
y(\theta)=\int_{0}^{2 \pi} K(\theta, \tau) z(\tau) d \tau .
$$

The function $F(\theta)$ has been defined in the form (14); consequently it can be expanded in the form of equation (13):

$$
F(\theta)=F_{1} \Phi_{1}(\theta)+F_{2} \Phi_{2}(\theta)+\cdots .
$$

Thus a simpler form of Eq. (9) is obtained:

$$
\Phi(\theta)=\frac{\lambda_{1}}{\lambda_{1}-\lambda} F_{1} \Phi_{1}(\theta)+\frac{\lambda_{2}}{\lambda_{2}-\lambda} F_{2} \Phi_{2}(\theta)+\cdots .
$$

Moreover, if the definition of $F(\theta)$ is substituted into Eq. (11), one has

$$
\begin{aligned}
F_{i} & =-\frac{\lambda}{4} \int_{0}^{2 \pi} \int_{0}^{2 \pi} K(\theta, \tau) \frac{g(\tau)}{[f(\tau)]^{1 / 2}} \Phi_{i}(\theta) d \tau d \theta \\
& =-\frac{1}{4} \frac{\lambda}{\lambda_{i}} \int_{0}^{2 \pi} \frac{g(\tau)}{[f(\tau)]^{1 / 2}} \Phi_{i}(\tau) d \tau ; \quad i=1,2, \cdots
\end{aligned}
$$

Hence, the solution of the lifting-line problem can be found immediately, in the form of a series expansion in eigenfunctions [Eq. (16)], where the coefficients are calculated from Eq. (17), provided, of course, that the eigenvalues and functions are known for the planform under consideration.

After discussing briefly the physical meaning of the eigenfunctions, we shall consider the problem of their numerical computation and present typical examples.

4. Physical interpretation. The preceding formulation has been largely mathematical. Although the theorems of the Hilbert-Schmidt theory tell us that the homogeneous Eq. (10) must have positive real eigenvalues, we have at present no insight into their physical significance. Returning to our original notation, we have

$$
\lambda=-4 / \pi l=-16 a / \pi(\mathrm{cm})_{0} .
$$


It is clear that positive eigenvalues correspond to negative values of the profile parameter $m_{0}$, which is the reference value of the two-dimensional lift slope. Now, the homogeneous problem [Eq. (10)] amounts to the determination of the circulation distribution of the wing in question when its angle-of-attack distribution $\alpha(y)$ is identically zero. We see that this problem has non-trivial solutions only for discrete negative values of the lift slope.

Physically, this result is easily understandable. The untwisted wing at zero incidence can produce lift only when its induced velocities (downwash) act to reinforce the circulation, and this can occur only when $m$ is negative! That this can occur only for certain discrete values also seems reasonable. The close analogy with the existence of selfreinforcing free vibrations of an elastic system at certain discrete natural frequencies is also obvious.

The eigenvalues are therefore the particular values of the parameter $\lambda$ for which the wing planform in question, with no twist and zero incidence, has non-vanishing lift distributions. The eigenfunctions represent these distributions.

5. Numerical calculation of eigenfunctions. So far, we have assumed implicitly that the eigenvalues and -functions were known, so that the solutions represented in Eqs. (16) and (17) could be carried out. We shall now consider the practical problem of their numerical determination. There are numerous methods available for this calculation,* and a few of them have been tried out on this problem. One that is used in vibration problems has been adopted, but no assurance can be given that it is the most accurate or the easiest.

In this procedure, we resort to the approximate expression of the $\Phi_{i}$ in finite trigonometric series. The numerical calculation of each $\Phi_{i}$ is then analogous to that proposed by Lotz $^{3}$ for the inhomogeneous case, but can be simplified by virtue of its homogeneity.

Returning to our original notation, as in Eq. (7), we have for the homogeneous case

$$
\varphi(\theta)=\lambda \int_{0}^{2 \pi} \log \left|\csc \frac{\theta-\tau}{2}\right| \varphi(\tau) f(\tau) d \tau .
$$

Let $\varphi(\theta)=\sum_{1} c_{n} \sin n \theta$ and $f(\theta)=\sum_{0} b_{n} \cos n \theta$. Then, using a known expression for the logarithmic kernel, we have

$$
\sum_{1} c_{n} \sin n \theta=\lambda \int_{0}^{2 \pi} \sum_{1}^{\infty} \frac{\sin m \theta \sin m \tau}{m} \sum_{1} c_{i} \sin j \theta \sum_{0} b_{k} \cos k \theta d \theta .
$$

Under suitable conditions of convergence, which are assumed here, this leads to a system of homogeneous linear equations for the $c_{n}$. The system can be solved approximately provided that the infinite series in Eq. (19) is terminated after the same number of terms as the other trigonometric series involved. This approximation leads to a matrix equation that can be solved numerically by a simple process of iterative multiplication: ${ }^{11}$

$$
\frac{2}{\pi}\{c\}=\lambda[k]\{c\},
$$

where $\{c\}$ is the column matrix of $c_{n}$ 's, and $[k]$ is the square matrix

*See, for example, Reference 10 and the references listed there. 


$$
\left[\begin{array}{llll}
2 b_{0}-b_{2} & b_{1}-b_{3} & b_{2}-b_{4} & \cdots \\
\left(b_{1}-b_{3}\right) / 2 & \left(2 b_{0}-b_{4}\right) / 2 & \left(b_{1}-b_{5}\right) / 2 & \cdots \\
\left(b_{2}-b_{4}\right) / 3 & \left(b_{1}-b_{5}\right) / 3 & \left(2 b_{0}-b_{6}\right) / 3 & \ldots \\
\ldots \ldots \ldots \ldots \ldots \ldots \ldots \ldots \ldots \ldots \ldots \ldots \ldots \ldots \ldots \ldots \ldots \ldots \ldots \ldots \ldots \ldots \ldots \ldots \ldots
\end{array}\right] .
$$

Numerical solution of Eq. (20) leads to an eigenvalue $\lambda_{1}$, and a corresponding matrix $\left\{c^{(1)}\right\}$, say, whose members are the Fourier constants of $\Phi_{1} /(f)^{1 / 2}$ or $\varphi_{1}(\theta)$, say $c_{1}^{(1)}$, $c_{2}^{(1)}, \ldots$. To determine the next eigenvalue and -function, the same numerical procedure is used, after eliminating the first set by one of the standard techniques, e.g. by use of the orthogonality relation

$$
\sum m c_{m}^{(i)} c_{m}^{(i)}=0 \quad \text { if } \quad i \neq j .
$$

This procedure has been employed to calculate approximately the eigenvalues and -functions of a widely-used family of planforms, namely the family of "trapezoidal" wings, whose chord length $c(y)$ varies linearly from $y=0$ to $y=2 a$. The results will be presented below.

6. Examples. A. Elliptic planform.

This case is represented by

$$
c m=(c m)_{0} \sin \theta
$$

or

$$
f=1 \text {. }
$$

Hence

$$
K(\theta, \tau)=\log \left|\csc \frac{\theta-\tau}{2}\right|,
$$

and the eigenvalues and -functions for this case are*

$$
\lambda_{n}=n / \pi ; \quad \Phi_{n}(\theta)=\pi^{-1 / 2} \sin n \theta .
$$

The solution of an inhomogeneous lift-distribution problem for an elliptical planform is therefore given by

$$
\begin{aligned}
\Phi(\theta) & =\varphi(\theta)=\Gamma / 2=4 a U \sum_{1} A_{n} \sin n \theta, \\
A_{n} & =\left(n+\frac{16 a}{(c m)_{0}}\right)^{-1} B_{n}, \\
B_{n} & \equiv \frac{2 / \pi}{U(c m)_{0}} \int_{0}^{\pi} g(\tau) \sin n \dot{\tau} d \tau=\frac{2}{\pi} \int_{0}^{\pi} \alpha \sin \tau \sin n \tau d \tau .
\end{aligned}
$$

These formulas are well known. ${ }^{12}$

B. Trapezoidal planform. The planforms of greatest practical interest, in view of their common use in aeronautics, are those represented by

*Cf. Reference 9, p. 130. 


$$
c m=(c m)_{0}\left\{1-t\left|\frac{y}{2 a}\right|\right\} ; \quad-2 a \leqq y \leqq 2 a
$$

or

$$
f=\frac{\sin \theta}{1-t|\cos \theta|} ; \quad 0 \leqq \theta \leqq \pi .
$$

where $t$ is a constant.

TABLE I

Eigenvalues for Trapezoidal Wings

\begin{tabular}{cccccc}
\hline \hline TAPER RATIO $T$ & 1 & 2 & 3 & 3.50 & 4.00 \\
\hline \hline$\lambda_{1}$ & .36726 & .29665 & .26553 & .25537 & .24722 \\
$\lambda_{2}$ & .8685 & .61731 & .51508 & .48303 & .45780 \\
$\lambda_{8}$ & 1.3512 & 1.0031 & .8480 & .7982 & .7585 \\
$\lambda_{4}$ & 1.8286 & 1.3470 & 1.1305 & 1.0614 & 1.0065 \\
$\lambda_{5}$ & 2.2950 & 1.7230 & 1.4504 & 1.3632 & 1.2944 \\
$\lambda_{6}$ & 2.7572 & 2.0728 & 1.7400 & 1.6336 & 1.5496 \\
$\lambda_{7}$ & 3.3209 & 2.4511 & 2.0900 & 1.9847 & 1.9008 \\
$\lambda_{8}$ & 3.8448 & 2.8165 & 2.3789 & 2.2568 & 2.1625 \\
$\lambda_{9}$ & 5.0487 & 4.4275 & 3.2631 & 2.9394 & 2.7021 \\
$\lambda_{10}$ & 5.7616 & 5.2158 & 3.7958 & 3.4008 & 3.1093 \\
\hline
\end{tabular}

\section{TABLE II}

\begin{tabular}{|c|c|c|c|c|c|}
\hline$c_{m}^{(n)}$ & $T=1$ & $T=2$ & $T=3$ & $T=3.5$ & $T=4.0$ \\
\hline $\begin{array}{l}c_{1}^{(1)} \\
c_{3}^{(1)} \\
c_{5}^{(1)} \\
c_{7^{(1)}} \\
c_{9}^{(1)}\end{array}$ & $\begin{array}{r}+.59990 \\
-.04933 \\
+.00072 \\
+.00053 \\
+.00028\end{array}$ & $\begin{array}{r}+.54354 \\
-.01387 \\
-.01100 \\
+.00028 \\
-.00094\end{array}$ & $\begin{array}{l}+.51446 \\
+.00644 \\
-.01279 \\
-.00066 \\
-.00119\end{array}$ & $\begin{array}{r}+.50375 \\
+.01368 \\
-.01278 \\
-.00110 \\
-.00132\end{array}$ & $\begin{array}{r}+.49525 \\
+.01974 \\
-.01247 \\
-.00147 \\
-.00145\end{array}$ \\
\hline $\begin{array}{l}c_{1}{ }^{(3)} \\
c_{3}^{(3)} \\
c_{5}^{(3)} \\
c_{7^{(3)}} \\
c_{9}{ }^{(3)}\end{array}$ & $\begin{array}{l}+.14955 \\
+.60194 \\
-.21574 \\
+.03634 \\
+.00082\end{array}$ & $\begin{array}{l}+.02992 \\
+.55224 \\
-.12402 \\
-.03257 \\
+.00511\end{array}$ & $\begin{array}{r}-.02691 \\
+.52148 \\
-.05528 \\
-.04809 \\
-.00068\end{array}$ & $\begin{array}{r}-.04616 \\
+.50782 \\
-.03100 \\
-.05003 \\
-.00371\end{array}$ & $\begin{array}{r}-.06183 \\
+.49537 \\
-.01099 \\
-.05020 \\
-.00635\end{array}$ \\
\hline $\begin{array}{l}c_{1}^{(5)} \\
c_{3}^{(5)} \\
c_{5}^{(5)} \\
c_{7}^{(5)} \\
c_{9}^{(5)}\end{array}$ & $\begin{array}{l}+.07860 \\
+.32339 \\
+.47680 \\
+.32639 \\
+.10075\end{array}$ & $\begin{array}{l}+.06207 \\
+.15086 \\
+.49449 \\
-.24570 \\
-.02524\end{array}$ & $\begin{array}{l}+.05736 \\
+.06048 \\
+.50181 \\
-.13417 \\
-.07544\end{array}$ & $\begin{array}{r}+.05714 \\
+.02879 \\
+.49618 \\
-.09390 \\
-.08500\end{array}$ & $\begin{array}{r}+.05760 \\
+.00268 \\
+.48866 \\
-.06070 \\
-.08925\end{array}$ \\
\hline $\begin{array}{l}c_{1}^{(7)} \\
c_{3}^{(7)} \\
c_{5}^{(7)} \\
c_{7}^{(7)} \\
c_{9}^{(7)}\end{array}$ & $\begin{array}{l}+.04596 \\
+.20074 \\
+.43372 \\
+.39752 \\
-.35774\end{array}$ & $\begin{array}{l}+.02561 \\
+.14254 \\
+.21269 \\
+.39032 \\
-.34917\end{array}$ & $\begin{array}{r}+.00600 \\
+.11266 \\
+.09220 \\
+.45196 \\
-.25385\end{array}$ & $\begin{array}{l}+.00043 \\
+.10890 \\
+.05589 \\
+.46896 \\
-.20937\end{array}$ & $\begin{array}{r}-.00365 \\
+.10816 \\
+.02895 \\
+.48042 \\
-.16527\end{array}$ \\
\hline $\begin{array}{l}c_{1}^{(9)} \\
c_{8}^{(9)} \\
c_{5}^{(9)} \\
c_{7}^{(9)} \\
c_{9}^{(9)}\end{array}$ & $\begin{array}{r}+.00815 \\
+.06094 \\
+.22312 \\
+.49177 \\
+.58651\end{array}$ & $\begin{array}{l}+.05221 \\
+.16744 \\
+.32499 \\
+.44450 \\
+.51959\end{array}$ & $\begin{array}{l}+.03918 \\
+.11292 \\
+.24314 \\
+.31287 \\
+.49923\end{array}$ & $\begin{array}{l}+.03555 \\
+.09307 \\
+.21699 \\
+.25698 \\
+.49608\end{array}$ & $\begin{array}{r}+.03294 \\
+.07569 \\
+.19688 \\
+.20307 \\
+.49456\end{array}$ \\
\hline
\end{tabular}

Fourier coefficients $c_{m}{ }^{(n)}$ of the functions $\varphi_{n}(\theta)$ (Odd values of $n$ ) 
TABLE III

Fourier coefficients $c_{m}{ }^{(n)}$ of the functions $\varphi_{n}(\theta)$ (Even values of $n$ )

\begin{tabular}{|c|c|c|c|c|c|}
\hline$c_{m}^{(n)}$ & $T=1$ & $T=2$ & $T=3$ & $T=3.5$ & $T=4.0$ \\
\hline $\begin{array}{l}c_{2}^{(2)} \\
c_{4}^{(2)} \\
c_{6}^{(2)} \\
c_{8}^{(2)} \\
c_{10}{ }^{(2)}\end{array}$ & $\begin{array}{r}+.62793 \\
-.13722 \\
+.01264 \\
+.00163 \\
+.00064\end{array}$ & $\begin{array}{r}+.54543 \\
-.07127 \\
-.01852 \\
-.00003 \\
-.00099\end{array}$ & $\begin{array}{r}+.50380 \\
-.03155 \\
-.02355 \\
-.00268 \\
-.00122\end{array}$ & $\begin{array}{l}+.48897 \\
-.01798 \\
-.02384 \\
-.00385 \\
-.00147\end{array}$ & $\begin{array}{r}+.47651 \\
-.00694 \\
-.02345 \\
-.00481 \\
-.00175\end{array}$ \\
\hline $\begin{array}{l}c_{2}{ }^{(4)} \\
c_{4}^{(4)} \\
c_{6}{ }^{(4)} \\
c_{8}{ }^{(4)} \\
c_{10}{ }^{(4)}\end{array}$ & $\begin{array}{r}+.25463 \\
+.54549 \\
-.28016 \\
+.06867 \\
-.00381\end{array}$ & $\begin{array}{r}+.11692 \\
+.52247 \\
-.19186 \\
-.02812 \\
+.00828\end{array}$ & $\begin{array}{r}+.04780 \\
+.50865 \\
-.10477 \\
-.05609 \\
+.00044\end{array}$ & $\begin{array}{r}+.02407 \\
+.49960 \\
-.07303 \\
-.06100 \\
-.00417\end{array}$ & $\begin{array}{r}+.00467 \\
+.49017 \\
-.04686 \\
-.06282 \\
-.00836\end{array}$ \\
\hline $\begin{array}{l}c_{2}{ }^{(6)} \\
c_{4}{ }^{(6)} \\
c_{6}{ }^{(6)} \\
c_{8}{ }^{(6)} \\
c_{10}{ }^{(6)}\end{array}$ & $\begin{array}{r}+.14045 \\
+.37005 \\
+.40287 \\
+.35825 \\
+.13026\end{array}$ & $\begin{array}{l}+.09593 \\
+.19703 \\
+.43699 \\
-.30691 \\
-.00235\end{array}$ & $\begin{array}{l}+.07476 \\
+.10285 \\
+.47565 \\
-.18484 \\
-.07443\end{array}$ & $\begin{array}{r}+.06899 \\
+.06753 \\
+.47671 \\
-.13793 \\
-.08973\end{array}$ & $\begin{array}{r}+.06557 \\
+.03825 \\
+.47605 \\
-.09959 \\
-.09808\end{array}$ \\
\hline $\begin{array}{l}c_{2}{ }^{(8)} \\
c_{4}{ }^{(8)} \\
c_{8}{ }^{(8)} \\
c_{8}{ }^{(8)} \\
c_{10}{ }^{(8)}\end{array}$ & $\begin{array}{r}+.07570 \\
+.24065 \\
+.45828 \\
+.35072 \\
-.36860\end{array}$ & $\begin{array}{r}+.06768 \\
+.17700 \\
+.24371 \\
+.32604 \\
+.38416\end{array}$ & $\begin{array}{r}+.03750 \\
+.12625 \\
+.11728 \\
+.41322 \\
-.29392\end{array}$ & $\begin{array}{r}+.03002 \\
+.11791 \\
+.07741 \\
+.43762 \\
-.25128\end{array}$ & $\begin{array}{r}+.02498 \\
+.11455 \\
+.04796 \\
+.45529 \\
+.20898\end{array}$ \\
\hline $\begin{array}{l}c_{2}{ }^{(10)} \\
c_{4}^{(10)} \\
c_{6}^{(10)} \\
c_{8}^{(10)} \\
c_{10}{ }^{(10)}\end{array}$ & $\begin{array}{r}+.00688 \\
+.06776 \\
+.24126 \\
+.50300 \\
+.58052\end{array}$ & $\begin{array}{r}+.09938 \\
+.22027 \\
+.36526 \\
+.46377 \\
+.49813\end{array}$ & $\begin{array}{r}+.07132 \\
+.15621 \\
+.27584 \\
+.34179 \\
+.47927\end{array}$ & $\begin{array}{l}+.06221 \\
+.13367 \\
+.24644 \\
+.29092 \\
+.47762\end{array}$ & $\begin{array}{r}+.05477 \\
+.11401 \\
+.22295 \\
+.24157 \\
+.47815\end{array}$ \\
\hline
\end{tabular}

The approximate calculation described above has been applied to this case.

It is important to notice that $f$ depends on the taper parameter $t$, but not on the aspect ratio. Consequently, the terms in Eq. (20) are independent of aspect ratio, and each set of eigenvalues and -functions can be used for an entire family of planforms having the same taper ratio.

The numerical results for this class of wings are summarized in Tables I-III and Figures 2-5. Figures 2 and 3 present the first ten eigenvalues as functions of the taper ratio $T$, which is defined as

$$
T \equiv \frac{(\mathrm{cm})_{0}}{(\mathrm{~cm})_{t i p}}=\frac{1}{1-t} .
$$

In Figures 4 and 5 are shown a typical set of eigenfunctions $\Phi_{n}$, namely the set corresponding to the case $T=1$ (rectangular wing), for $n=1$ to 10 .

In Tables II and III are presented the values of the first ten Fourier coefficients $c_{n}^{(m)}$ of the functions $\varphi_{m}(\theta)$, for $m=1$ to 10 and for several taper ratios between 1 and 4. It will be recalled that these functions are related to the normalized eigenfunctions by the relation

$$
\Phi_{m}(\theta)=\varphi_{m}(\theta)[f(\theta)]^{1 / 2} .
$$

These tables include all of the numerical data required to use the present method of solution with trapezoidal planforms within the range of values of $T$ indicated.

7. Formulas for practical application (rigid wings). In this section we shall present 


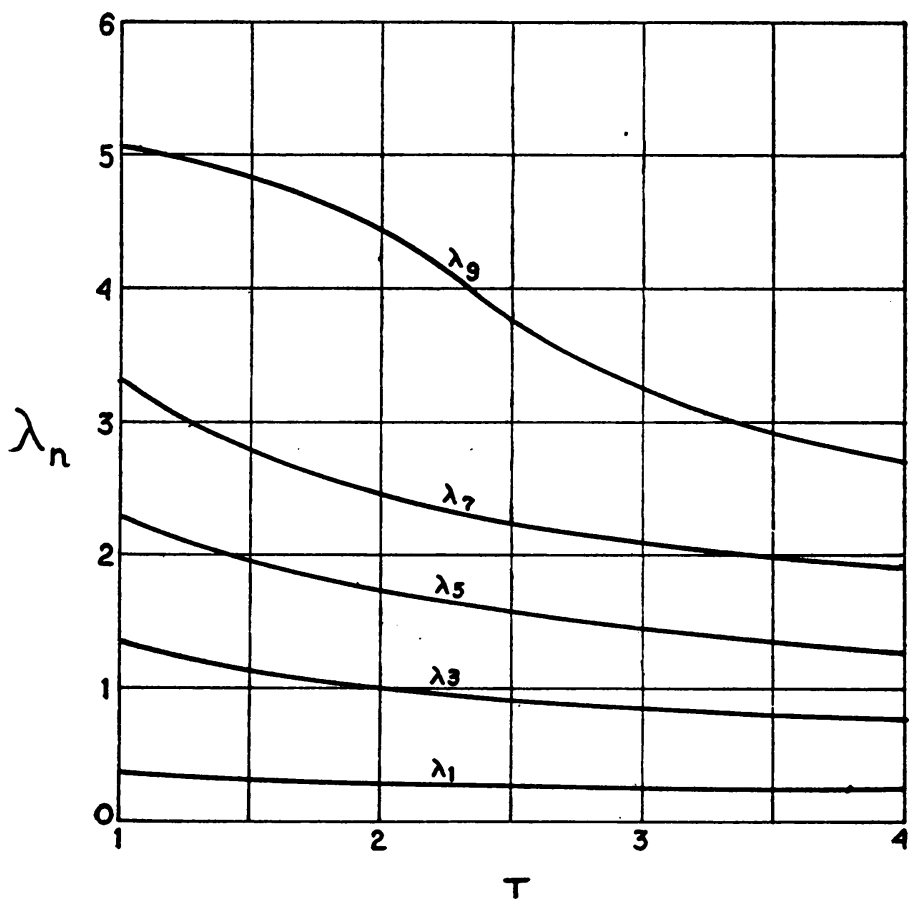

Fig. 2. Eigenvalues $\lambda_{n}$ for trapezoidal wings of taper ratio $T$. Odd values of $n$.

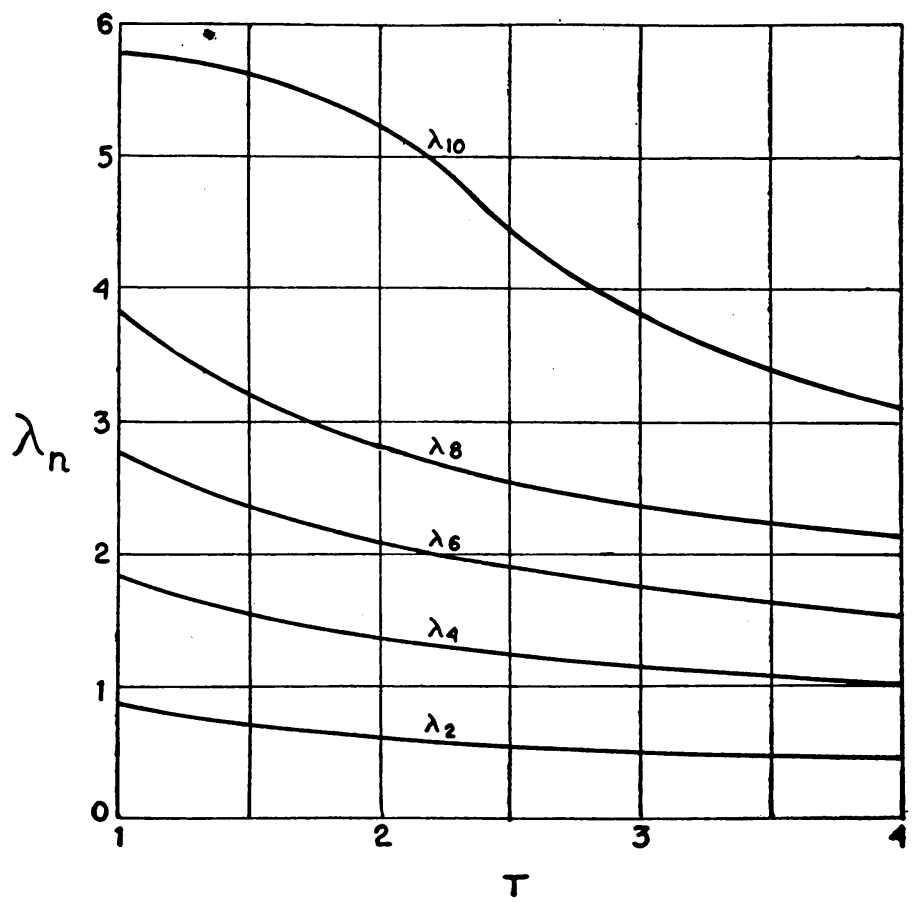

Fig. 3. Eigenvalues $\lambda_{n}$ for trapezoidal wings of taper ratio $T$. Even values of $n$. 


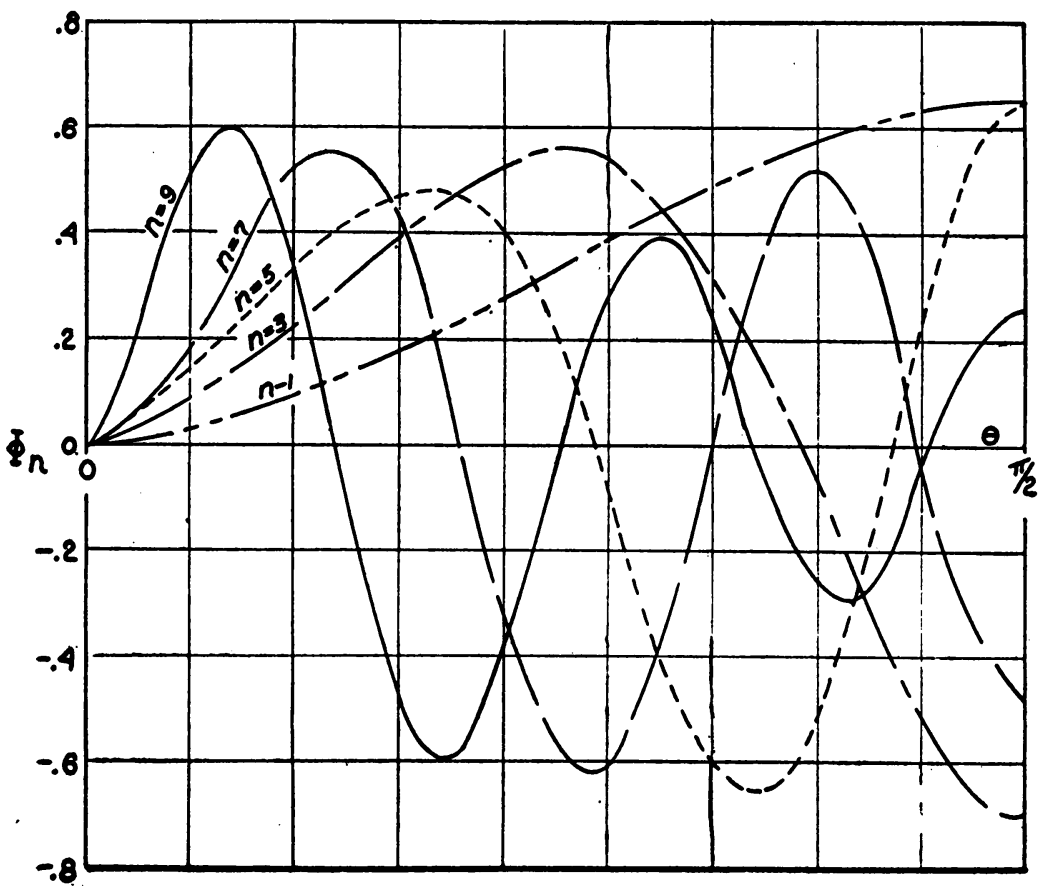

Fig. 4. The eigenfunctions $\Phi_{n}(\theta)$ for rectangular wings. Odd values of $n$.



Fig. 5. The eigenfunctions $\Phi_{n}(\theta)$ for rectangular wings. Even values of $n$. 
certain formulas that may be convenient in the practical application of the present theory to the classical problem of the Prandtl wing theory, i.e. to calculate the forces and the spanwise distribution of lift on a prescribed rigid wing.

It has already been found, in Eqs. (16) and (17), that

$$
\Phi(\theta)=4 U a \sum a_{n} \Phi_{n}(\theta),
$$

where

$$
a_{n} \equiv-\frac{1}{16 U a} \frac{\lambda}{\lambda_{n}-\lambda} \int_{0}^{2 \pi} g(\tau) \varphi_{n}(\tau) d \tau .
$$

Thus, the circulation distribution is given by

$$
\Gamma=2 \varphi=2 \Phi / f^{1 / 2}=8 U a \sum a_{n} \varphi_{n}(\theta) .
$$

When, as in the case of the trapezoidal wings above, the functions $\varphi_{n}$ are given in terms of their Fourier constants, it is convenient to calculate the coefficients $a_{n}$ as follows:

$$
\begin{aligned}
Q_{n} & =-\frac{1}{16 U a} \frac{\lambda}{\lambda_{n}-\lambda} \int_{0}^{2 \pi} g(\tau) \sum_{1} c_{m}^{(n)} \sin m \tau d \tau \\
& =-\frac{\pi}{16 U a} \frac{\lambda}{\lambda_{n}-\lambda} \sum_{1} c_{m}^{(n)} B_{m},
\end{aligned}
$$

where $B_{m} \equiv(1 / \pi) \int_{0}^{2 \pi} g(\tau) \sin m \tau d \tau$; i.e. the Fourier coefficient of the angle-of-attack function $g$.

Moreover, the usual sine series for $\Gamma$ may be useful; it is commonly written as

$$
\Gamma=8 U a \sum_{1} A_{n} \sin n \theta .
$$

It is seen that the two "Fourier" coefficients, for expansion in sines and in eigenfunctions, respectively, are related as follows:

$$
A_{n}=\sum_{1} c_{n}^{(m)} \mathrm{Q}_{m} .
$$

Hence, certain formulas of the Prandtl theory ${ }^{2,3,5,12}$ can be transformed immediately to our new formulation:

$$
\begin{aligned}
& C_{L} \equiv \text { (Coefficient of total lift) }=\pi A \sum_{1} c_{1}^{(m)} Q_{m} \\
& \begin{aligned}
C_{l} \equiv \text { (Coefficient of total rolling moment) }=-\frac{\pi}{4} A \sum_{1} c_{2}^{(m)} a_{m}, \\
C_{D} \equiv \text { (Coefficient of induced drag) }=\pi A \sum_{m} \sum_{n} a_{m} Q_{n} \sum_{k} k c_{k}^{(m)} c_{k}^{(n)} \\
=\pi A \sum_{1} \lambda_{m} Q_{m}^{2},
\end{aligned}
\end{aligned}
$$

where $A$ denotes the aspect ratio, a constant.

Other useful expressions can be obtained by the well-known expedient of dividing the circulation distribution into so-called "basic" and "additional" parts, which are 
independent of $C_{L}$ and of $g(\theta)$, respectively. These formulas will not be developed here, since they are quite analogous to those employed in other methods of calculating spanwise load distributions. ${ }^{13}$

An additional simplification occurs in most practical cases because the planform is symmetrical about its center-line $y=0$. This makes $f(\theta)$ symmetrical about $\theta=\pi / 2$, and it is then found that $\varphi_{1}, \varphi_{3}, \varphi_{5}, \cdots$ are symmetrical while $\varphi_{2}, \varphi_{4}, \cdots$ are antisymmetrical; thus half of the coefficients $c_{m}^{(n)}$ are identically zero. The trapezoidal planforms of the preceding section provide an example of this. In this situation it is clear that symmetrical and antisymmetrical lift-distribution problems are independent of one another; i.e., symmetrical $\alpha(y)$ 's will produce only symmetrical $\Gamma(y)$ 's, and antisymmetrical $\alpha(y)$ 's will produce only antisymmetrical $\Gamma(y)$ 's. Obviously, practical problems involving arbitrary $\alpha(y)$ 's will usually be handled by considering the symmetrical and anti-symmetrical parts of $\alpha(y)$ separately.

In summary, it may be said that, for rigid wings, the use of eigenfunctions reduces every lift-distribution calculation to the same simplicity that ordinarily occurs only for the elliptical planform. However, since the eigenfunctions are not conveniently tabulated and are not as simple as trigonometric functions, this simplification is partly illusory, and for practical use one actually works with the Fourier constants $c_{n}^{(m)}$. Nevertheless, the simplification represented by Eqs. (25), (26), and (27) is very great; one calculates the span loading $\Gamma$ by a straightforward process requiring only the Fourier coefficients of the angle-of-attack function $g$, besides tabulated quantities. No procedure of successive approximations is required.

8. Formulas for practical application (elastic wings). In the case of the elastic wing, whose ultimate geometrical configuration is determined by the airload distribution, it appears that the present theory may offer rather important possibilities. At present, calculations for such wings are either made without attention to the aerodynamical principles of the Prandtl theory-i.e. by neglecting the effects of the trailing vortex sheet entirely - or by assuming special and simple geometrical and elastic properties, which may be completely inapplicable to wings encountered in practical engineering problems. Another method is to perform a process of successive approximations, estimating the elastic deflection from the rigid-wing airloads, repeating the rigid-wing calculation for the deflected wing, and so forth. It is clear that this process must fail at each of the so-called divergence speeds, where the elastic restoring force in some mode of deflection becomes insufficient to resist the air forces. It is also likely that the convergence of the process becomes poor as such a divergence situation is approached, and that this will make it impossible either to predict the divergence speed or to calculate air loads with satisfactory accuracy near such a speed.

To attack this problem, we first note explicitly that the load distribution $\rho U \Gamma(y)^{*}$ is linearly related to the angle-of-attack distribution $\alpha(y)$ or $g(\theta)$, according to the Prandtl theory considered here. Let us denote by $\odot[g]$ the circulation distribution of a given wing at the speed $U$ when the angle-of-attack function is $g(\theta)$. Thus $\mathscr{P}[g]$ is the "Prandtl functional" calculated in the preceding paragraphs, i.e.

$$
\Gamma=\odot[g]
$$

and it is linear in $g$; i.e.

${ }^{*} \rho$ denotes the fluid density. 


$$
\begin{aligned}
\mathcal{P}\left[g_{1}+g_{2}\right] & =\mathcal{P}\left[g_{1}\right]+\mathcal{P}\left[g_{2}\right], \\
\odot[k g] & =k \odot[g] .
\end{aligned}
$$

Now if the subscripts $r$ and $e$ denote, respectively, the contributions due to the built-in angle of attack, $\alpha_{r}(y)$, and the elastic deflection under load $\alpha_{\bullet}(y)$, we have

$$
\begin{aligned}
\alpha(y) & =\alpha_{r}(y)+\alpha_{\bullet}(y), \\
g(\theta) & =g_{r}(\theta)+g_{\bullet}(\theta), \\
\Gamma=\mathcal{P}[g]= & \mathcal{P}\left[g_{r}\right]+\mathcal{P}\left[g_{e}\right] .
\end{aligned}
$$

Moreover, the elastic deflection (twist) is determined by a linear functional involving the load distribution $\rho U \Gamma(y)$. Ordinarily, it is satisfactory to calculate this deflection by means of the torsional influence function $F(y, \eta)$, which denotes the angular twist at $y$ due to a concentrated unit torque at $\eta$. This function can be calculated or it can be determined experimentally; for practical airplane wings it is often a very irregular function. It is used to calculate the elastic twist as follows:

$$
\alpha_{\bullet}(y)=\int_{0}^{2 a} F(y, \eta) \frac{d J}{d \eta} d \eta,
$$

where $J(y)$ is the torque about the elastic axis at any spanwise station $y$ :

$$
\frac{d \mathrm{~J}}{d y}=M+\rho U \mathrm{r} e,
$$

where $M=M(y) \equiv$ aerodynamic moment about aerodynamic center at $y$

$$
=\left(\frac{1}{2}\right) \rho U^{2} c^{2} c_{m}(y)
$$

$c_{m}(y) \equiv$ moment coefficient of the profile at $y$ (a given function),

$$
e=e(y) \equiv \text { distance between aerodynamic center and elastic axis at } y \text { (a given }
$$
function).

Thus

$$
\alpha_{\bullet}(y)=\frac{1}{2} \rho U^{2}\left\{\int_{0}^{2 a} F(y, \eta) c^{2} c_{m} d \eta+2 \int_{0}^{2 a} F(y, \eta) e \odot[g / U] d \eta\right\}
$$

or

$$
\begin{aligned}
g_{0} / U= & (c m)_{0} \alpha_{e} \sin \theta \\
= & \frac{1}{2} \rho U^{2}(c m)_{0} \sin \theta\left\{\int_{0}^{2 a} F(y, \eta) c^{2} c_{m} d \eta\right. \\
& \left.\quad+2 \int_{0}^{2 a} F(y, \eta)\left(\odot\left[\frac{g_{r}}{U}\right]+\odot\left[\frac{g_{e}}{U}\right]\right) e d \eta\right\} .
\end{aligned}
$$

It should be noticed that there are two terms on the right-hand side of Eq. (32) involving only known functions and a third involving the elastic deflection. Equation (32) is a linear integral equation of second kind, but involves a very complicated kernel. Its solution might be found in terms of the eigenvalues and -functions of the homo- 
geneous case, i.e. the case $c_{m}=g_{r}=0$. These eigenvalues would represent the values of $\frac{1}{2} \rho U^{2}(\mathrm{~cm})_{0}$ for which wing deflection would occur with no initial moment or angle of attack; viz., the divergence speeds.

For practical calculation, using the present technique of handling the Prandtl problem, we make use of a symbolism that is equivalent to Eqs. (6), (10), or (18):*

$$
2 \varphi_{n}(\theta)=\odot\left[4 \frac{\lambda-\lambda_{n}}{\lambda} f \varphi_{n}\right]
$$

or

$$
P\left[f \varphi_{n}\right]=\frac{\lambda / 2}{\lambda-\lambda_{n}} \varphi_{n} .
$$

where $f^{1 / 2} \varphi_{n}$ is the normalized eigenfunction, as before. This shows, incidentally, that the unique property of the eigenfunctions can alternatively be expressed thus: $f \varphi_{n}$ is the angle-of-attack distribution that produces, on the given wing, a circulation distribution proportional to $\varphi_{n}$.

Therefore, if we expand $g_{r} / U$ and $g_{0} / U$ in series of $\varphi_{n}$ 's, we can immediately operate term-by-term with the Prandtl functional symbol $\beta$; i.e. if

$$
\frac{g_{r}}{U}=f(\theta) \sum b_{i}^{(r)} \varphi_{i}(\theta) \quad \text { and } \quad \frac{g_{e}}{U}=f(\theta) \sum b_{i}^{(e)} \varphi_{i}(\theta),
$$

then

$$
\mathcal{P}[g / U]=\sum\left(b_{i}^{(r)}+b_{i}^{(o)}\right) \mathcal{P}\left[f \varphi_{i}\right]=\sum\left(b_{i}^{(r)}+b_{i}^{(o)}\right) \frac{\lambda / 2}{\lambda-\lambda_{i}} \varphi_{i} .
$$

But according to Eqs. (13),

$$
\begin{aligned}
b_{n}^{(e)}= & \int_{0}^{2 \pi} \varphi_{n}(\theta) \frac{g_{e}}{U} d \theta \\
= & \frac{1}{2} \rho U^{2}(c m)_{0}\left\{\int_{0}^{2 \pi} \varphi_{n}(\theta) \sin \theta \int_{0}^{2 a} F(y, \eta) c^{2} c_{m} d \eta d \theta\right. \\
& \left.+\sum_{i}\left(b_{i}^{(r)}+b_{i}^{(e)}\right) \frac{\lambda}{\lambda \dot{-} \lambda_{i}} \int_{0}^{2 \pi} \varphi_{n}(\theta) \sin \theta \int_{0}^{2 a} F(y, \eta) e \varphi_{i} d \eta d \theta\right\}
\end{aligned}
$$

(for $n=1,2, \cdots$ ).

This equation has the form

$$
b_{n}^{(0)}=\frac{1}{2} \rho U^{2}(c m)_{0}\left\{C_{n}+D_{n}+\sum_{i} E_{n i} b_{i}^{(0)}\right\} .
$$

${ }^{*}$ For, according to equation (6), the symbolism $2 \varphi=\mathcal{P}[g]$ means

$$
4 f \varphi+(4 / \pi \lambda) \partial \varphi / \partial r=g
$$

in any case. But for the eigenfunctions, or rather the related $\varphi_{n}$,

hence

$$
4 f \varphi_{n}+\left(4 / \pi \lambda_{n}\right) \partial \varphi_{n} / \partial r=0 ;
$$

$$
4 f \varphi_{n}+(4 / \pi \lambda) \partial \varphi_{n} / \partial r=4\left(1-\lambda_{n} / \lambda\right) f \varphi_{n} .
$$


where the terms have the following physical meanings:

$C_{n}=n$th "Fourier" coefficient, for expansion in $\varphi_{n}(\theta)$ 's, corresponding to the twist due to the aerodynamic-center moments $c_{m}(y)$, at unit dynamic pressure.

$D_{n} \equiv \sum^{p} E_{n i} b_{i}^{(r)}=n$th "Fourier" coefficient corresponding to the twist due to the rigid-wing airload distribution, at unit dynamic pressure.

$\sum_{i} E_{n i} b_{i}^{(e)}=n$th "Fourier" coefficient corresponding to the twist due to the airload resulting from elastic twist, at unit dynamic pressure.

Moreover, $E_{n i}$ is equal to $\left[\lambda /\left(\lambda-\lambda_{i}\right)\right] F_{n i}$, where $F_{n i}$ is the $n$th "Fourier" coefficient for the series expansion of the twist produced by the circulation distribution $\varphi_{i}$, at unit dynamic pressure.

It is clear that the elastic-wing problem is solved when the $b_{n}^{(e)}$ are determined from Eqs. (36), since they define the only unknown function, the elastic deflection under load. It is seen that the essential calculation required for the elastic wing is the determination of a set of $F_{n i}$ 's, i.e., one must calculate the elastic deflection under a loading proportional to each $\varphi_{i}$ for a sufficient number of values of $i$, and must then determine the "Fourier" coefficients of these deflections. To do this, it is most convenient to use the formula

$$
F_{n i}=\sum_{m} c_{m}^{(n)} \int_{0}^{2 \pi} \Theta_{i}(\theta) \sin \theta \sin m \theta d \theta
$$

where

$$
\Theta_{i}(\theta) \equiv \int_{0}^{2 a} F(y, \eta) e \varphi_{i} d \eta
$$

Equations (36) are most conveniently solved by standard matrix methods. ${ }^{14}$ Of the several techniques available, the partial-fraction type of expansion* gives the most engineering information, since the latent roots, which appear as simple poles in the expansion, correspond to the divergence speeds of the wing, as has been mentioned above.

Once again it will be found that, for a symmetrical planform, the symmetrical and antisymmetrical distributions are independent of one another. Thus a set of odd-numbered and a set of even-numbered latent roots appear, corresponding respectively to divergence in symmetrical and antisymmetrical modes, and so forth.

In summary, the following may be said regarding the present treatment of the problem of the elastic wing. The integral equation of the problem, Eq. (32), has been put into matrix form, and an essential feature of the treatment is the proposal that matrix techniques be used for numerical solution. Moreover, in making this formulation, essential use has been made of the knowledge of a complete set of functions, the eigenfunctions of the planform, for which the Prandtl functional can be written down immediately.

9. Acknowledgements. The use of the eigenfunctions of the Prandtl equation, as well as the physical interpretation of these functions, was suggested in 1939 by Professor Theodore von Kármán. Following his suggestion, the present author worked out the details while he was a staff member of the California Institute of Technology. The eigenvalues and -functions for trapezoidal planforms were calculated by $\mathrm{H}$. Fischer in

${ }^{*}$ See, for example, reference 14, pp. 78, 134-141. 
1940 and later by H. T. Nagamatsu, both of the California Institute. This method was used extensively by the engineering staff of Northrop Aircraft, Inc., and the application to elastic wings was worked out at Northrop by the author and H. R. Lawrence in 1943-44. The formulation in terms of matrices and the application of matrix techniques in practical computations are largely due to Mr. Lawrence.

Incidentally, it may be mentioned that Mr. Lawrence's work has progressed somewhat farther than the material presented here. He has extended the calculations, in practical form, to problems of aileron reversal, wing deflection and divergence in accelerated flight, and other similar cases. It is expected that his results along these lines will be published soon.

\section{REFERENCES}

1. L. Prandtl, Tragflügeltheorie, Nachrichten d. K. Gesellschaft d. Wiss. zu Göttingen, Math.-phys. Klasse 1918, 451-477. Reprinted in Vier Abhandlungen zur Hydrodynamik und Aerodynamik, Göttingen, 1927, or Edwards Bros., 1943.

2. H. Glauert, Elements of the aerofoil and airscrew theory, Cambridge, 1926.

3. I. Lotz, Berechnung der Auftriebsverteilung beliebig geformter Flügel, Zeitschr. Flugtechn. Motorluftschiffahrt 22, 189-195 (1931).

4. H. Multhopp, Die Berechnung der Auftriebsverteilung von Tragflügeln, Luftfahrtforschung 15, 153-169 (1938).

5. E. Trefftz, Prandtlsche Tragflächen- und Propellertheorie, Zeitschr. angew. Math. Mech. 1, 206-218 (1921).

6. K. Schröder, Die Prandtlsche Integro-differentialgleichung, Abh. Preussischen Akad. Wiss. 1939, Nr. 16 (35 pp.).

7. H. Gebelein, Über die Integralgleichung der Prandtlschen Tragflächentheorie, Ing.-Arch. 7, 297-325 (1936).

8. P. Frank and R. von Mises, Die Differential- und Integralgleichungen der Mechanik und Physik, (2nd edition) Vieweg, Braunschweig, 1930, or Rosenberg, New York, 1943.

9. R. Courant and D. Hilbert, Methoden der Mathematischen Physik I (2nd edition) Springer, Berlin, 1931, or Interscience, New York, 1943.

10. L. Collatz, Genäherte Berechnung von Eigenwerten, Zeitschr. angew. Math. Mech. 19, 224-249; 297-318 (1939).

11. Th. von Kármán and M. A. Biot, Mathematical méthods in engineering (Chapter V), McGraw Hill, New York, 1940.

12. M. Munk, Fluid dynamics for aircraft designers, Ronald, New York, 1929, pp. 99-102.

13. R. F. Anderson, Determination of the characteristics of tapered wings, N. A. C. A. Technical Report No. 572 (1936).

14. R. A. Frazer, W. J. Duncan, and A. R. Collar, Elementary matrices and some applications to dynamics and differential equations, Cambridge University Press, Cambridge, 1938. 